FACULDADE DE CIÊNCIAS ECONÔMICAS DA UFRGS Análise
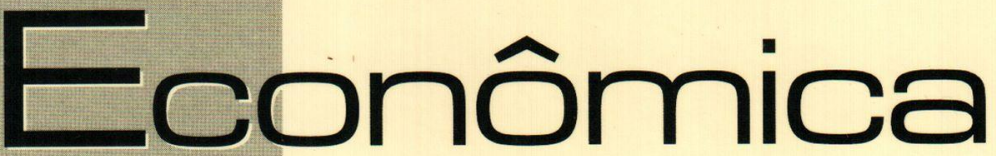

FLUTUACÃO CAMBIAL E METAS INFLACIONÁRIAS EM

ECONOMIAS EMERGENTES

OTAVIANO CANUTO E MárCIO HOLLAND

ATEORIA DE FORMACĆOO DE PRECOS E A TEORIA DOS PREÇOS DE

PRODUCAO

LOIZALBERTO ESTEVES

AECONOMIA EVOLUCIONISTA: UM CAPITULOSISTEMMICO DA

TEORIA ECONOMICA?

HUGOE. A. DA GAMACERQUEIRA

RELAÇ̃O INFLAÇÃO-PRODUTO NO BRASIL NO PERIODO PÓS-

REAL

MARCOSC. HOLANDA

DIVIIDA PÚBLICAMOBILLÍRIA FEDERAL BRASILEIRA: HISTÓRIA RECENTE E PERSPECTIVAS EXPLOSIVAS

ROGÉRIO MEURER E ROBERT WAYNE SAMOHYI

A CEPAL EA INTEGRACÃO REGIONAL LATINO-AMERICANA JACQUELINE A. HERNANDEZ HAFFNER

POPPER, HAYEK EA (IM)POSSIBILIDADE DE PREDIÇŌES ESPECIFICAS EM CIEENCIAS SOCIAIS

BRENA PAULA MAGNO FERNANDEZ

DINÂMICA RECENTE DO PROCESSO DE INCUBACG̃O DE

EMPRESAS DE BASE TECNOLOGICA NO BRASIL

EDUARDO GONÇALVES

TRIBUTACĀO COM SACRIF́CIO EQUUITATIVO: O CASO DO IMPOSTO DE RENDA PESSOA FISICA

STEFANO FLORISSI E EDUARDO PONTUAL RIBEIRO

A NOVATEORIA DO IMPÉRIOE AS VELHAS TEORIAS DO

IMPERIALISMO

GENTIL CORAZZA

Ô ABREALAS - A NOVA INSERÇÃO DO BRASIL NA ECONOMIA MUNDIAL

EMLLLANO LUIS KLEN

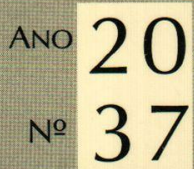

Março, 2002 
UNIVERSIDADE FEDERAL DO RIO GRANDE DO SUL

Reitora: Profa. Wrana Maria Panizzi

FACULDADE DE CIÊNCIAS ECONÔMICAS

Diretor: Prof. Pedro Cézar Dutra Fonseca

CENTRO DE. ESTUDOS E PESQUISAS ECONÔMICAS

Diretor: Prof. Gentil Corazza

DEPARTAMENTO DE CIÊNCIAS ECONÔMICAS

Chefe: Prof. Luiz Alberto Oliveira Ribeiro de Miranda

DAPARTAMENTO DE CIÊNCIAS CONTÁBEIS E ATUARIAIS

Chefe: João Marcos Leão da Rocha

CURSO DE PÓS-GRADUAÇÃO EM ECONOMIA

Coordenador: Prof. Eduardo Pontual Ribeiro

CURSO DE PÓS-GRADUAÇÃO EM ECONOMIA RURAL

Coordenador: Prof Jalcione Almeida

CONSELHO EDITORIAL: Carlos G. A. Mielitz Netto (UFRGS), Eduardo A. Maldonado Filho (UFRGS), Eduardo P. Ribeiro (UFRGS), Eleutério F. S. Prado (USP), Eugênio Lagemann (UFRGS), Fernando Cardim de Carvalho (UFRJ), Fernando Ferrari Filho (UFRGS), Fernando de Holanda Barbosa (FGV/RJ), Flávio Vasconcellos Comim (UFRGS), Gentil Corazza (UFRGS), Giácomo Balbinotto Neto (UFRGS), Gustavo Franco (PUC/RJ), Jan A. Kregel (Università di Bologna e John Hopkins University), João Rogério Sanson (UFSC), Joaquim Pinto de Andrade (UnB), Jorge Paulo Araújo (UFRGS), Juan H. Moldau (USP), Marcelo S. Portugal (UFRGS), Maria Alice L.ahorgue (UFRGS), Paul Davidson (University of Tennessee), Paulo Dabdab Waquil (UFRGS), Pedro Cézar Dutra Fonseca (UFRGS), Philip Arestis (South Bank University), Roberto C. Moraes (UFRGS), Ronald Otto Hillbrecht (UFRGS), Sabino da Silva Porto Jr. (UFRGS), Stefano Florissi (UFRGS), Werner Baer (Univ, of Illinois at Urbana-Champaign).

COMISSÃO EDITORIAL: Eduardo Augusto Maldonado Filho, Fernando Ferrari Filho, Gentil Corazza, Marcelo Savino Portugal, Paulo Dabdab Waquil; Roberto Camps Moraes.

EDITOR: Fernando Ferrari Filho

EDITOR ADJUNTO: Gentil Corrazza

SECRETÁRIA: Vanessa Hoffmann de Quadros

REVISÃO DE TEXTOS: Vanete Ricacheski

FUNDADOR: Prof. Antônio Carlos Santos Rosa

Os materiais publicados na revista Análise Econômica são da exclusiva responsabilidade dos autores. É permitida a reprodução total ou parcial dos trabalhos, desde que seja citada a fonte. Aceita-se permuta com revistas congêneres. Aceitam-se, também, livros para divulgação, elaboração de resenhas e recensões. Toda correspondência, material para publicação (vide normas na terceira capa), assinaturas e permutas devem ser dirigidos ao seguinte destinatário:

PROF FERNANDO FERRARI FILHO

Revista Análise Econômica - Av. João Pessoa, 52 CEP 90040-000 PORTO ALEGRE - RS, BRASIL Telefones: (051) 316-3348 e 316-3440 - Fax: (051) 316-3990

E-mail: rae@vortex ufrgs br

Análise Econômica

Ano 20, n 37 , março, 2002 - Porto Alegre

Faculdade de Ciências Econômicas, UFRGS, 2000

Periodicidade semestral, março e setembro.

1. Teoria Econômica - Desenvolvimento Regional Economia Agrícola - Pesquisa Teórica e Aplicada -

Periódicos. I. Brasil.

Faculdade de Ciências Econômicas,

Universidade Federal do Rio Grande do Sul.

CDD 330.05

CDU 33 (81) (05) 


\section{Dinâmica recente do processo de incubação de empresas de base tecnológica no Brasil}

Eduardo Gonçalves*

Resumo: As fases iniciais de uma nova firma inovadora são caracterizadas por numerosos desafios e considerável incerteza. Para aumentar as chances de sobrevivência das novas empresas de base tecnológica as incubadoras têm importante papel. Neste artigo é realizada uma revisão da literatura sobre inovação e papel dos empreendedores. Algumas características das pequenas empresas de base tecnológica são apresentadas, com base numa amostra de incubadoras brasileiras. $\mathrm{O}$ artigo proporciona informação sobre a qualificação profissional dos empreendedores, bem como o número de pesquisadores com titulação de doutorado envolvidos com o processo de criação de firmas. As principais estratégias competitivas e inovativas das empresas também são discutidas. Ao lado do crescimento do número de incubadoras tem havido um processo de concentração na Região Sudeste e Sul do Brasil. Na parte final do trabalho, alguns desafios e soluçōes para o fortalecimento do processo de incubação de empresas no Brasil são discutidos.

Palavras-Chave: Empresas de Base Tecnológica, Incubadoras, Brasil.

Abstract: The initial stages of an innovative firm are characterized by numerous challenges and considerable uncertainty. Technology incubators play an important role because they increase the chances of success of the new technology-based firms. First, this paper reviews the literature on innovation and the role of entrepreneurs. Second, it presents some characteristics of small technology-based firms based on an sample of Brazilian technology incubators. It provides information about the professional qualifications of the entrepreneurs as well as the number of professionals who have a $\mathrm{PhD}$ that are involved in the process of starting a firm. The main competitive and innovative strategies of the new technology-based firms are discussed too. Moreover, the growth in the number of technology incubators, that is concentrated in the southeastern states of Brazil, is showed. Finally, a new light is thrown on some challenges and solutions to strengthen the process of incubation in Brazil.

Key words: Technology-Based Firms, Technology Incubators, Brazil. 
A introdução no mercado de novos produtos e processos ou a melhoria significativa dos já existentes é cercada por incerteza e riscos, uma vez que não há controle perfeito sobre o resultado do uso de insumos durante o esforço inovativo. Embora seja atributo do empreendedor ligar as idéias novas ao mercado, um conjunto de obstáculos afeta os estágios iniciais de uma firma inovadora, comprometendo a geraçāo de renda, emprego e difusão de novas tecnologias.

Segundo a OECD (1997), além de problemas associados ao fluxo de caixa, há custos de entrada, custos fixos, falta de acesso ao mercado de capital, insuficiente informação técnica e sobre o mercado e pouca habilidade gerencial dos empreendedores. Nesse sentido, as incubadoras possuem papel fundamental para aumentar as chances de sobrevivência de novas empresas inovadoras e garantir os benefícios da inovação para a sociedade.

Segundo informações da Associação Nacional de Entidades Promotoras de Empreendimentos de Tecnologias Avançadas - ANPROTEC (2000), cerca de $87 \%$ das incubadoras brasileiras consideram muito importante o incentivo ao empreendedorismo. O desenvolvimento econômico regional, o desenvolvimento tecnológico, a geração de empregos e diversificação econômica regional é objetivo muito importante para, respectivamente, $75 \%, 71 \%, 58 \%$ e $42 \%$ das incubadoras. Apenas 7\% deram importância muito alta para a meta de auferir lucro.

Este trabalho possui o objetivo de aperfeiçoar a compreensão sobre o papel das incubadoras no apoio aos empreendedores que criam empresas de base tecnológica e a distribuição regional destas iniciativas no Brasil. Os empreendedores serão caracterizados quanto à formação profissional e à titulação e serão destacados os tipos de estratégias competitivas e inovativas seguidas pelas empresas de base tecnológica para superar obstáculos que envolvem o processo inovativo. $\mathrm{O}$ artigo identifica os principais desafios do processo de incubação de empresas, além de discutir possíveis soluçōes. As evidências empíricas estão principalmente baseadas em questionários respondidos por um conjunto de empresas vinculadas a incubadoras de base tecnológica brasileiras.

A próxima seção apresenta uma resenha teórica sobre a inovação e o papel dos empreendedores, além de descrever os objetivos do processo de incubação de empresas. A base de dados é explicada na seção 3, que antecede as seções que procuram revelar o perfil profissi- 
onal e a qualificação dos empreendedores e algumas estratégias usadas pelas empresas de base tecnológica. A distribuição regional das incubadoras brasileiras e os desafios do processo de incubação são discutidos nas seçōes 6 e 7 . A seção 8 conclui o trabalho.

\section{Referencial teórico}

\subsection{Inovações e empreendedores}

As inovações diferem das invenções pelo sucesso comercial, pois "enquanto não forem levadas à prática, as invenções são economicamente irrelevantes" (SCHUMPETER, 1982, p. 62).

Como destacam MONCK et al. (1988), inovação inclui invenção e muitos outros estágios de implementação como pesquisa, desenvolvimento, produção e comercialização. De acordo com DOSI (1988), inovação diz respeito à busca, à descoberta, à experimentação, ao desenvolvimento, à imitação e à adoção de novos produtos, novos processos de produção e novos sistemas organizacionais.

Não obstante o tratamento pioneiro do tema por Schumpeter, há críticas relacionadas à ausência de atenção adequada às condições prémercado do desenvolvimento tecnológico, ao focar a inovação como nova combinaçāo de fatores. Segundo HAGEDOORN (1996), isso resultou não só na abstração das fases iniciais da tecnologia como na negligência das mudanças tecnológicas menores (não radicais). Ou seja, mudanças técnicas baseadas em rotinas existentes foram consideradas irrelevantes.

Nesse sentido, HAGEDOORN (1996) destaca que contribuições mais recentes complementaram a teoria da inovação schumpeteriana ao introduzirem as noções de learning-by-doing e outras formas de aprendizado organizacional. Essas idéias mostraram que as firmas desenvolvem conhecimento através de processos mais amplos que apenas PED. Embora capacitação tecnológica esteja relacionada intimamente com capacitação em PESD, vários modos de adquirir novo conhecimento estão fora do PẺD formal (MALECKI, 1991).

Por outro lado, contribuição fundamental foi dada por Schumpeter ao definir o papel do empreendedor na introdução da inovação. Segundo SCHUMPETER (1982, p. 48)

... as inovações no sistema econômico não aparecem, via de regra, de tal maneira que primeiramente as novas necessidades surgem espontaneamen- 
te nos consumidores e então o aparato produtivo se modifica sob sua pressão. Não negamos a presença desse nexo. Entretanto, é o produtor que, via de regra, inicia a mudança econômica, e os consumidores são educados por ele, se necessário; săo, por assim dizer, ensinados a querer coisas novas, ou coisas que diferem em um aspecto ou outro daqueles que tinham o hábito de usar."

Longa controvérsia estabeleceu-se sobre a origem das inovações, a partir das noções de science ou technology push ou de demand-pull. Numa, as descobertas científicas promovem inovações em tempo e direção lineares ${ }^{1}$. Noutra, a demanda de mercado puxa inovações dos laboratórios empresariais (MALECKI,1991). FREEMAN \& SOETE (1997) destacam que enquanto alguns cientistas enfatizaram fortemente o elemento de pesquisa original e invençāo, negligenciando o mercado, economistas tenderam a sobrelevar o lado da demanda, com argumentos do tipo: "necessidade é a mãe da invenção". De fato, a grande maioria das inovações situam-se entre os dois extremos, precisando de algum grau de combinação entre possibilidades de novas técnicas è de mercado.

Isso porque, de um lado, é preciso reconhecer a necessidade, ou seja, o mercado potencial para novos produtos e processos. Por outro lado, a inovação requer conhecimento técnico, que pode estar disponível ou necessitar de novo conhecimento científico e tecnológico, resultante de atividade de pesquisa original. As tentativas de associar possibilidades técnicas ao mercado envolvem desenvolvimento experimental, design, testes e marketing e, por causa de sua complexidade, refletiram-se na profissionalização da $\mathrm{P} \mathscr{}$ D industrial, processo constatado no decorrer do século XX. A importância do empreendedor reside justamente em ligar idéias novas ao mercado (FREEMAN $\mathcal{G}$ SOETE, 1997), o que não constitui tarefa fácil.

O caráter de incerteza técnica da inovação e a possibilidade de erro na avaliação do futuro mercado e da competição representam

\footnotetext{
${ }^{1}$ A abordagem que ressalta as inovações como um processo interativo entre usuário e produtor (LUNDVALL, 1988) permite entender o processo inovativo a partir da interação entre os agentes e não através de uma relação linear. Nesse sentido, o "modelo linear" da inovação perde importância. Como esclarece MALECKI (1991) tal modelo sugere uma seqüência unidirecional de etapas: pesquisa básica e aplicada, desenvolvimento do produto e processo, produção e difusão e comercialização. Como enfatizam MONCK et al. (1988) as noções science push ou market pull implicam uma relação monocausal ou linear. É muito mais plausível a existência de uma relação bidirecional entre ciência e tecnologia. Ex: desenvolvimento da máquina a vapor promoveu e precedeu ao entendimento científico dos princípios e leis da termodinâmica.
} 
obstáculos a uma tentativa bem sucedida de inovar. O processo inovativo é marcado pelo caráter aleatório, acidental e arbitrário, que surge da complexidade do relacionamento entre ciência avançada, tecnologia e mudança de mercado. "The uncertainty lies in the extent to which the innovation will satisfy a variety of technical criteria without increased cost of development, production or operation" (FREEMAN \& SOETE, 1997, p. 243). A natureza da incerteza que envolve a inovação exerce forte incentivo para a firma não empreender um tipo mais radical de inovaçāo de produto e para concentrar a PED sobre inovações defensivas, imitadoras, diferenciação de produto e inovação de processo. Quanto mais radical uma inovação maior o grau de incerteza envolvido.

Dessas considerações, FREEMAN $\mathcal{G}$ SOETE (1997) destacam três características ligadas à possibilidade de sucesso da firma inovadora:

1) As novas descobertas e possibilidades técnicas criadas pelo avanço na pesquisa científica permitem à firma que monitora a fronteira científica ser a primeira capaz de aproveitar a nova possibilidade da inovação. Intensos gastos de PED permitirão transformar esse conhecimento em vantagens competitivas;

2) Uma firma atenta às necessidades de seus clientes está em melhores condiçōes de identificar mercados potenciais para idéias novas e fontes de insatisfação do consumidor, que pode conduzir a melhorias de produtos e processos;

3) Ligar as possibilidades técnicas às de mercado a partir da conjugação dos fluxos de informação e de novas idéias.

As propriedades fundamentais do processo inovativo são apropriadamente resumidas por DOSI (1988), através de cinco fatos estilizados:

i) Incerteza: os resultados dos esforços inovativos dificilmente podem ser conhecidos ex ante. A atividade inovativa é empreendida por agentes motivados pela busca de lucro, os quais possuem percepções de oportunidades técnicas e econômicas ainda não exploradas. Entretanto, o caráter incerto da atividade inovativa advém da existência de problemas tecno-econômicos cuja solução é desconhecida e da impossibilidade de precisar as conseqüências das ações, ao invés de ausência de informação relevante sobre a ocorrência de eventos conhecidos.

ii) as novas oportunidades tecnológicas possuem crescente dependência dos avanços no conhecimento científico, o que é uma importante propriedade da inovação contemporânea. No século XX, a inovação foi capaz de extrair novas oportunidades de avanços científicos (da termodinâmica a biologia, eletricidade, física quântica, mecânica etc.); 
iii) crescente complexidade das atividades inovativas e de pesquisa, o que favoreceu as organizaçōes formais ao invés de inovadores individualmente isolados;

iv) o processo de aprendizado pode ocorrer também através de learning by doing e learning by using, em que os agentes aprendem como usar, melhorar e produzir bens mediante o processo de fazê-los, atividades informais de resolução de problemas relativos à produção, solução das necessidades específicas dos clientes e superação de vários tipos de gargalos técnicos;

v) o padrāo de mudança tecnológica não pode ser descrito como reações simples e flexíveis às mudanças de mercado. Isso se deve a três fatos: 1) a direção da mudança técnica é ditada pelo estado da arte das tecnologias em uso; 2) a própria natureza da tecnologia determina o grau de ajuste de produtos e processos às mudanças de condições eco-

nômicas; 3 ) a probabilidade de conduzir avanço tecnológico depende do nível já alcançado por cada agente (firmas, organização e países), ou seja, a mudança é técnica e cumulativa.

\subsection{O papel das incubadoras na criação de empresas inovativas}

Uma incubadora de base tecnológica é um mecanismo institucional que busca fortalecer a criação, o desenvolvimento e a consolidação de pequenas empresas inovadoras, oferecendo infra-estrutura técnica, operacional e outros serviços aos empreendedores (MEDEIROS, 1996; MEDEIROS \& ATAS, 1995).

Os serviços, que permitem explorar e comercializar o resultado de pesquisa e estabelecer a empresa, podem ser divididos da seguinte forma (OECD, 1997): i) infra-estrutura física e acesso a laboratórios de universidades; ii) suporte administrativo, representado por desenvolvimento de habilidades comerciais e gerenciais, realização de estudos de marketing e assistência na comercialização; iii) suporte técnico, através de acesso a facilidades técnicas internas, programas de transferência de tecnologia e assistência de pessoal qualificado; iv) acesso ao financiamento, devido à intermediação entre a empresa e as fontes de financiamento; $v$ ) assistência jurídica e proteção da propriedade intelectual; vi) estabelecimento e fortalecimento de uma rede de inter-relações e de cooperação entre os agentes do processo inovativo.

Como ressaltam MACULAN 8 CARLEIAL (1999), o primeiro objetivo de uma empresa, ao instalar-se na incubadora, é o acesso aos recursos materiais e humanos a custos reduzidos. A oferta de infra-estrutura 
administrativa e técnica a baixo custo opera como um fator que compensa a carência de fontes de financiamento para pequenas empresas ou a necessidade de elevado volume de capital inicial. O segundo é a reunião de competências, embora a formação e qualificação profissional dos empresários já sejam uma vantagem dos empreendedores para a capacitação tecnológica da empresa. O período de incubação é comparado com um período de aprendizado.

Uma das principais competências a ser conquistada pelo empreendedor é a gerencial e comercial, dado que os empresários possuem como origem o ambiente universitário, no caso de professores, ou são recém formados, como na maioria das vezes. Ou seja, embora a capacidade de inovação seja elevada no plano tecnológico e produtivo, é limitada no plano comercial. A qualificação dos empreendedores facilita as interações e a inovação, pois estão familiarizados com as atividades de pesquisa e são capazes de learning by searching. $O$ desenvolvimento de novos produtos/processos é uma atividade central, além de haver forte interação com pesquisadores externos. Para isso, as empresas precisam estabelecer relaçōes com diversos parceiros, como: universidades, centros de $\mathrm{P} \mathcal{B} \mathrm{D}$, empresas usuárias e fornecedoras, agências governamentais, gerência da incubadora e outras empresas incubadas.

LEMOS 8 MACULAN (1998a) avaliaram os principais motivos dos empreendedores para ingresso em incubadoras, com base numa amostra de 53 pequenas empresas de 12 incubadoras de base tecnológica no Brasil. Quatro motivaçōes foram consideradas: infra-estrutura material e administrativa a baixo custo; vínculo formal com a universidade; formação gerencial; e intermediação com agências de apoio à inovação. Do total de respostas, $34 \%$ apontaram a infra-estrutura material e administrativa a baixo custo como razão principal para a incubação, o que se explica pela escassez de recursos financeiros que caracteriza as pequenas empresas. Desse modo, é possível priorizar os recursos no desenvolvimento tecnológico dos produtos e serviços e alocar o reduzido número de funcionários que possuem nas atividades principais das empresas. $\mathrm{O}$ apoio administrativo concedido pela incubadora desobriga os profissionais das atividades rotineiras. O vínculo formal com a universidade foi considerado o segundo aspecto mais importante para os empresários (27\%). A expectativa nesse caso está relacionada com o apoio tecnológico e institucional, que permitem trocas de informações relevantes e obtenção de recursos junto a outras entidades.

A formação gerencial propiciada aos empreendedores, incluindo consultoria, cursos, legalização da empresa, apoio na comercialização 
e interação com outras empresas de base tecnológica, foi o terceiro item a receber maior número de respostas (19\%). A intermediação com agências de apoio tinha menor importância para os empresários (7\%), o que pode revelar desconhecimento dos empresários quanto à existência de programas de apoio a essas empresas no país, como os do CNPq, FINEP e SEBRAE.

Com base na mesma amostra, LEMOS 8 MACULAN (1998b) avaliaram que 40 das 53 empresas pesquisadas enfrentavam dificuldade de financiamento. Trinta e quatro tinham problemas na transformação de seus projetos em produtos e serviços e 34 encontravam dificuldades para colocar os produtos no mercado. Muitas dessas dificuldades são amenizadas pelo papel da incubadora ao apoiar a obtenção de financiamento, as atividades de produção e a comercialização. No caso do financiamento, só $14 \%$ das empresas afirmaram que as incubadoras não contribuíam para superar esse tipo de obstáculo.

É fundamental a função de intermediação entre as empresas e as instituições governamentais, pois as principais fontes de recursos financeiros citadas pelas empresas correspondem aos recursos públicos oriundos de programas da FINEP e CNPq, como bolsas de desenvolvimento tecnológico ( $30 \%$ das respostas). As outras fontes, em ordem de importância segundo o número de respostas, são: financiamento junto a órgãos públicos (20\%); recursos próprios (15\%); atração de capital de risco (12\%); prestação de serviços (9\%); empréstimos junto a bancos privados (6\%); venda de produtos (3\%); e outras fontes $(5 \%)$.

As principais contribuições das incubadoras para que as empresas superem obstáculos produtivos sāo: consultoria e orientaçāo (39\%) e espaço físico e infra-estrutura $(21 \%)^{2}$. No que se refere à dificuldade de comercialização, a oferta de assessoria de marketing e o apoio na divulgação do produto foram considerados as principais contribuições da incubadora.

Entretanto, nem todos os serviços são oferecidos pelas incubadoras num grau adequado. Pesquisa realizada em 1996 por BAÊTA (1999) revela que os empresários apontaram como menos satisfatórios a consultoria na área de marketing e a obtenção de "capital semente".

\footnotetext{
${ }^{2}$ Nesse caso, os empresários foram questionados sobre o "apoio das incubadoras na superação das dificuldades de produção", especificamente. A ordem de importância das principais contribuições foi estabelecida a partir de 33 respostas extraídas de 29 pequenas empresas de base tecnológica. Essa é uma base amostral distinta da questão que avaliou as "motivações das pequenas empresas de base tecnológica para ingresso em incubadoras", que contou com 121 respostas de questionários respondidos por 53 empresas.
} 
Para a permanência das empresas na incubadora, foram considerados essenciais: os serviços de escritório, os cursos de treinamento, o acesso a laboratórios e as informações tecno-científicas, bem como os mecanismos de financiamento para equipamentos e bolsas. Os resultados divergentes de BAETA (1999) e LEMOS 8 MACULAN (1998b) no que se refere à assessoria em marketing podem estar refletindo diferenças amostrais ou um aperfeiçoamento dos serviços prestados pelas incubadoras no período decorrido entre as duas pesquisas. Isso porque LEMOS 3 MACULAN (1998b) ressaltaram que a partir de 1996 o Programa de Competitividade e Difusão Tecnológica do CNPq apoiou a implantação de núcleos de marketing nas incubadoras.

Mesmo assim, as vantagens do sistema de incubação de empresas refletem-se na redução da taxa de mortalidade desse tipo de empreendimento. MEDEIROS $\mathcal{S}$ ATAS (1995) registraram uma taxa de $40 \%$, contra evidências internacionais de 30\%. Das 166 empresas incubadas, registradas em sua pesquisa de 1996, BAÊTA (1999) destaca que houve apenas 9 casos de desistência de empreendedores. Estimativas recentes da ANPROTEC mostram que a taxa média de mortalidade é de $20 \%$ (MORTALIDADE..., 2000), mesmo percentual informado pela OECD (1997) para firmas de incubadoras norte-americanas após os cinco primeiros anos, na década de 1990.

O apoio do setor público a esses empreendimentos justifica-se pela existência de falhas de mercado que cria desincentivos à criação de empresas. Isso porque os empreendedores enfrentam obstáculos no início do negócio como: altos custos fixos e custos de entrada, falta de acesso ao capital acionário, informações de mercado e técnicas insuficientes e fracas habilidade gerenciais. Alem disso, a fase de start-up está associada a considerável incerteza e a problemas de fluxo de caixa. Outros gargalos podem afetar a firma após a fase de start-up, como o financiamento, além do gerenciamento e marketing não adequados estarem associados com taxa de mortalidade das novas firmas.

Quanto maior a incerteza associada com a tecnologia, maiores os riscos inerentes a novos empreendimentos. Portanto, os serviços da incubadora permitem reduzir tal incerteza e, conseqüentemente, aumen tar a probabilidade de sucesso. Além disso, as incubadoras podem ser vistas também como meio de conseguir retornos crescentes do gasto de PẺ público através da promoção da comercialização e difusão da tecnologia gerada (OECD, 1997). 


\section{Descrição da base de dados}

Os dados deste trabalho estão baseados numa pesquisa realizada em 2000, envolvendo 43 incubadoras de base tecnológica de 31 cidades diferentes (Quadro 1). Este número é elevado ao se considerar que naquele ano a ANPROTEC registrava 76 incubadoras de base tecnológica ${ }^{3}$, do total de 135 incubadoras do país.

Dentre as 148 empresas participantes da pesquisa, 84 indicaram apenas uma área de atuação. Destas, $43 \%$ atuavam no desenvolvimento de softuare. Outras 6 pertenciam ao setor eletro-eletrônico, 5 vinculavam-se ao ramo de comércio eletrônico e internet, 5 ofereciam serviços especializados, 4 eram do setor alimentos, 3 de automaçāo, 3 de mecânica, 2 de biotecnologia e 2 de telecomunicações. Outros setores, como por exemplo consultoria empresarial, novos materiais, química fina, energia e higiene possuíam uma empresa cada.

Entretanto, é comum que uma empresa atue em diferentes segmentos ao mesmo tempo. O Quadro 2 revela quais são as áreas de atuação mais indicadas pelas empresas das incubadoras, que são software, serviços especializados, eletro-eletrônica, automação, consultoria empresarial, hardware, telecomunicações, biotecnologia e comércio eletrônico/internet. O maior peso relativo dos setores vinculados à ciência da computação é coerente com as informações da ANPROTEC (2000), que destacam este setor como o principal entre as empresas residentes em incubadoras no Brasil.

De modo geral, as empresas que ingressam numa incubadora são muito heterogêneas, seja pela diversidade setorial em que atuam ou pelo estágio de desenvolvimento em que se encontram (MACULAN $\mathcal{6}$ CARLEIAL, 1999). O ingresso de uma empresa numa incubadora pode coincidir com sua criação, casos em que o empreendedor cria uma empresa para explorar um resultado de pesquisa. Em outros casos, a empresa já existente no mercado se aproxima da incubadora para aperfeiçoar tecnicamente um produto já existente ou para lançar novo produto, situação em que o projeto de PED é conduzido na incubadora.

Por causa dos diferentes estágios de desenvolvimento das empresas nem todas faturam nos anos iniciais de incubação. De um total de 147 empresas que informaram este campo, 97 já haviam colocado o

${ }^{3}$ Além das incubadoras de base tecnológica, há incubadoras tradicionais e mistas. Estas abrigam empresas de base tecnológica e também de ramos tradicionais como couro, confecçóes etc. 
produto/serviço no mercado após a entrada na incubadora. Outras 50 informaram que o produto/serviço ainda estava sendo desenvolvido. Entretanto, deste subtotal 12 faturavam. Esta receita deve estar associada à comercialização de outras linhas de produtos, muitas vezes de menor conteúdo tecnológico que o produto principal, usada para fomentar o processo de PËD deste. Portanto, cerca de 109 empresas já faturavam, mesmo ainda em processo de incubação.

\begin{tabular}{|c|c|c|}
\hline Incubadora & Cidade & $\begin{array}{l}\text { Número de } \\
\text { participantes }\end{array}$ \\
\hline BIOMINAS & Belo Horizonte/MG & 3 \\
\hline BIORIO & Rio de Janeiro/RJ & 6 \\
\hline BLUSOFT & Blumenau/SC & 9 \\
\hline CDT/UnB & Brasilia/DF & 4 \\
\hline CEFET-RJ & Rio de Janeiro/RJ & 4 \\
\hline CEI & Porto Alegre/RS & 2 \\
\hline CELTA & Florianópolis/SC & 1 \\
\hline CIATEC-NADE & Campinas/SP & 9 \\
\hline CIETEC & São Paulo/SP & 8 \\
\hline CIENTEC & Porto Alegre/RS & 1 \\
\hline COMPETE & Salvador/BA & 4 \\
\hline CRITT & Juiz de Fora/MG & 4 \\
\hline FUNARBEUFV & Viçosa/MG & 1 \\
\hline GÊNESIS-PUC & Rio de Janeiro/RJ & 8 \\
\hline IET & Novo Hamburgo/RS & 2 \\
\hline IETEC & Passo Fundo/RS & 2 \\
\hline IETEC & Porto Alegre/RS & 2 \\
\hline IEBTEC & Nova Friburgo/RJ & 1 \\
\hline IIES & Curitiba/PR & 4 \\
\hline INATEL & S. R Sapucai/MG & 1 \\
\hline INETEC & Uberlândia/MG & 3 \\
\hline INCUBATEP & Recife/PE & 11 \\
\hline INSOFT-CE & Fortaleza/CE & 3 \\
\hline INSOFT-BH & Belo Horizonte/MG & 2 \\
\hline INTEC & Curitiba/PR & 3 \\
\hline ITEBE & Betim/MG & 4 \\
\hline ITEC & Caxias do Sul/RS & 4 \\
\hline ITS & S. Mateus do Sul/PR & 3 \\
\hline ITSM & Santa Maria/RS & 1 \\
\hline MIDI & Joinville/SC & 10 \\
\hline MIDIVILLE & Joinville/SC & 3 \\
\hline NIT & Natal/RN & 1 \\
\hline $\begin{array}{l}\text { NUTEC } \\
\text { PADETEC }\end{array}$ & $\begin{array}{l}\text { Fortaleza/CE } \\
\text { Fortaleza/CE }\end{array}$ & $\begin{array}{l}3 \\
1\end{array}$ \\
\hline PIEBT & Belém/PA & 4 \\
\hline POLOVALE & S J dos & 2 \\
\hline Não identificada & Salvador/BA & 1 \\
\hline SOFTEX & Campinas/SP & 3 \\
\hline SOFTEX & Salvador/BA & 2 \\
\hline TECNOPARK & Rio do Sul/SC & 2 \\
\hline UFF & Niterói/RJ & 1 \\
\hline UNITEC-UNISINOS & Sāo Leopoldo/RS & 3 \\
\hline Não identificada & Nāo identificada & 2 \\
\hline \multicolumn{2}{|l|}{ TOTAL } & 148 \\
\hline
\end{tabular}

\section{Quadro 1: Origem das empresas de base tecnológica pesquisadas}

Fonte: dados da pesquisa. 


\begin{tabular}{|lr|lr|}
\hline Software & 75 & Equipamentos Médico-Oftalmológicos & 2 \\
Serviços Especializados & 25 & Optoeletrônica & 1 \\
Eletro-eletrônica & 18 & Química de Base & 1 \\
Automação & 16 & Química Fina & 1 \\
Consultoria Empresarial & 15 & Otica & 1 \\
Hardware & 14 & Tecnologia da Informação \\
Telecomunicações & 12 & Óleo Vegetal & 1 \\
Biotecnologia & 10 & Instrumentação & 1 \\
Comércio eletrônico/Internet & 9 & Construção civil & 1 \\
Mecânica & 6 & Óleo Vegetal & 1 \\
Farmacêutica & 5 & Ótica & 1 \\
Novos Materiais & 4 & Consultoria em Redes de Comunicação & 1 \\
Alimentos & 4 & Tecnologia da Informação \\
Cosméticos & 3 & Higiene bucal & 1 \\
Energia & 2 & Treinamento & 1 \\
Design & 2 & Compilação e Distribuição de Software r & 1 \\
\hline
\end{tabular}

Quadro 2: Setores de atuação mais indicados pelas empresas participantes da pesquisa

Obs: Respostas Múltiplas.

Fonte: dados da pesquisa.

\section{Perfil dos empreendedores, titulação e apoio de pesquisadores externos}

A Tabela 1 revela que, do total de 360 pessoas envolvidas no processo de criação das empresas de base tecnológica que participaram da pesquisa, $32 \%$ dos empreendedores eram formados em engenharia. A outra área de formação mais importante era a de Ciências da Computação, envolvendo aproximadamente $20 \%$ do total. Se forem somadas as proporçōes das pessoas formadas nas áreas mais diretamente associadas à geração de novas tecnologias (engenharias, ciências da computação, química, física, farmácia, ciências biológicas e agronomia), encontrar-se-á o total de $65 \%$. A formação nestas áreas e o elevado grau de escolaridade, comum entre este tipo de empreendedor, explica-se pela necessidade de conhecimento de princípios tecno-científicos básicos.

Nem todos empreendedores possuem elevado grau de titulação. Dos 360 pesquisados, 76 tinham titulação de mestrado e outros 34 eram doutores. O nível de qualificação do empreendedor é importante porque define a capacidade de inovação deste tipo de firma, bem como o conteúdo tecnológico do produto a ser desenvolvido. Além disso, quan- 
to maior a qualificação maior será o grau de proximidade com as atividades de $\mathrm{P} \mathcal{G} \mathrm{D}$, mais fácil ocorrerão as interações com outras instituições de ensino e pesquisa e melhor será a assimilação das diversas formas de aprendizado, cuja lista tem expandido muito nos últimos anos (MALECKI, 1991), como learning by doing, learning by using, learning by operating, learning by searching, learning by trying, learning by interacting, learning by selling, learning by failing.

Tabela 1: Perfil profissional dos empreendedores

Fonte: dados da pesquisa.

\begin{tabular}{l|lc}
\hline Formação & $\mathrm{N}$ & $\%$ \\
\hline Engenharias & 116 & 32,22 \\
Ciências da Computação & 71 & 19,72 \\
Administração & 23 & 6,39 \\
Química & 17 & 4,72 \\
Física & 14 & 3,89 \\
Ciências Econômicas & 9 & 2,50 \\
Arquitetura & 9 & 2,50 \\
Farmácia & 8 & 2,22 \\
Ciências Biológicas & 7 & 1,94 \\
Direito & 6 & 1,67 \\
Agronomia & 2 & 0,56 \\
Outros & 37 & 10,28 \\
Superior Incompleto & 28 & 7,78 \\
Nivel Médio & 13 & 3,61 \\
\hline Total & 360 & 100 \\
\hline
\end{tabular}

Como nem todos criadores das empresas possuem titulação de doutorado ou mestrado, as seguintes estratégias são adotadas pelas empresas de base tecnológica: 1) aproveitamento de vantagens associadas ao vínculo a uma incubadora, como suporte técnico, assistência de pessoal qualificado, programa de transferência de tecnologias e interrelações entre agentes. O ambiente da incubadora propicia às firmas algumas formas de aprendizado citadas acima, como o learning by interacting $e$ o learning by selling; 2 ) estabelecimento de uma rede de inter-relações com pesquisadores externos à empresa, que possuem alta qualificação ou titulação. Esta participação pode ocorrer via sociedade na empresa ou através de apoio informal, representando uma forma de reduzir os elevados custos fixos de PGD, aumentar a capacidade de aprendizado e de gerar inovações bem sucedidas tecnicamente.

Do total de 148 empresas que participaram da pesquisa, cerca de $15 \%$ possuíam pelo menos um fundador com titulação de doutorado, enquanto que em $25 \%$ dos casos havia pelo menos um fundador com 
mestrado. Destes últimos, havia apoio externo de pesquisadores doutores em $23 \%$ das empresas. Ou seja, 9 das 40 empresas fundadas por um mestre tinham apoio externo de pessoa com titulação superior. Oitenta e seis empresas não tinham fundador com mestrado ou doutorado, embora a grande maioria tivesse escolaridade superior. Em doze casos, havia apoio externo de doutores. Em resumo, 29\% das empresas (43 das 148 empresas) tinham auxílio de pesquisador doutor, seja fundador da empresa ou colaborador externo formal ou informal.

\section{Estratégias competitivas e de inovação das empresas de base tecnológica}

Questionados quanto à principal força dos seus produtos/serviços para competir, os empreendedores indicaram "ser diferenciado, não tendo similar no mercado". Esta estratégia recebeu $44 \%$ das respostas ou 37 das 85 empresas que informaram esse campo. A segunda principal estratégia de competição seguida pelas empresas, que recebeu $24 \%$ das respostas, foi colocar no mercado um produto que tem como vantagem o fato de "ser melhor adaptado a uma (algumas) aplicação(s) específica(s)". Os itens "seu menor preço, em relação aos concorrentes", "seu melhor desempenho técnico, em relação aos concorrentes" e "sua melhor qualidade" receberam respectivamente 14\%, 13\% e 6\% das respostas.

A ordem de importância das estratégias competitivas das empresas é coerente com a própria origem das empresas de base tecnológica, que visualizaram uma oportunidade tecnológica, transformando resultados de pesquisa acadêmica em produtos/serviços. A produção de um bem diferenciado, sem similar no mercado, ou a de um bem melhor adaptado a aplicações específicas (67\% dos casos) revela que este tipo de pequena empresa atua em nichos de mercado, que possuem menores barreiras à entrada.

Nestes casos, um dos principais desafios a serem enfrentados pelos empreendedores é justamente criar demanda para seu produto diferenciado, caso não existam clientes potenciais. Isto envolve recursos humanos e financeiros não disponiveis em condições satisfatórias nas empresas de base tecnológica, que podem ser, contudo, oferecidos pelas incubadoras. Particularmente, é fundamental o apoio gerencial e de comercialização do produto (marketing) com vistas a tornar bem sucedida a tentativa de associar a criação de um produto diferenciado, a 
partir da visualização de possibilidades técnicas, à sua aceitação pelo mercado.

A pequena importância relativa da competição via preço explicase pelo fato de que, nos ramos industriais de alta tecnologia, a principal estratégia competitiva ocorre por meio de diferenciação de produto quando inovações incrementais são geradas ou até mesmo via criação de bens que possuem impactos significativos sobre a estrutura produti$\mathrm{va}$, inovações radicais.

Adotando a classificação de FREEMAN 3 SOETE (1997), as estratégias de inovação das empresas de base tecnológica brasileiras podem variar entre ofensiva, defensiva e dependente ${ }^{4}$. Segundo os autores, as novas firmas baseadas em tecnologia são uma categoria especial de inovador ofensivo, atuando em nichos de mercado muito especializados. A estratégia ofensiva visa conquistar liderança técnica e de mercado através da introdução de novos produtos antes dos concorrentes. Esta estratégia requer forte participação do seu departamento de PGD para gerar conhecimento e informação científica e técnica não disponivel para a firma, além de outras formas de aprendizado. A introdução deste tipo de inovação requer também grande capacidade de design, construção e teste de protótipos e plantas piloto, além da proteção por patentes.

A sustentação da estratégia ofensiva necessita de elenco variado de competências que podem superar as que existem na maioria das pequenas empresas de base tecnológica residentes em incubadoras brasileiras. Outras estratégias podem ser comuns neste tipo de empresa brasileira. A estratégia defensiva pode requerer tanto esforço de P\&D quanto a ofensiva, mas não implica riscos de ser o primeiro a inovar e pode ter vantagens de evitar erros cometidos por inovadores pioneiros ou de lucrar com um mercado já estabelecido. Este tipo de inovador realiza melhoramentos em produtos/serviços já existentes, o que se relaciona com a estratégia de diferenciação de produto de muitos oligopólios. Esta pode ser a estratégia que melhor se enquadra nos casos em que os empreendedores afirmaram que seu principal produto apresentava melhor desempenho técnico, era melhor adaptado a algumas aplicações específicas ou tinha melhor qualidade.

\footnotetext{
${ }^{4}$ Em alguns casos, é possivel que a estratégia imitadora seja também usada por estas empresas. A única estratégia de FREEMAN $\mathcal{G}$ SOETE (1997) que não se adequa ao tipo de empresa aqui considerado é a tradicional, uma vez que as firmas que a adotam operam sob condições próximas à concorrência perfeita e praticamente não usam insumos como conhecimento científico.
} 
$\mathrm{Na}$ estratégia dependente quaisquer tentativas de iniciar ou imitar mudanças técnicas nos produtos são tomadas apenas a partir das necessidades de firmas das quais são satélites ou subordinadas. Para o caso das empresas residentes em incubadoras brasileiras, esta estratégia pode emergir quando: 1) grandes companhias realizam alianças e acordos com as menores, as quais passam a desenvolver produtos para as maiores; 2) as maiores empresas financiam projetos de PED ou subcontratam as pequenas empresas de base tecnológica, transformando-as em fornecedores especializados de PGD; 3) há aquisição das empresas incubadas ou ocorre a criação de uma empresa a partir de um empregado qualificado, sob estímulo de seu ex-empregador, para fornecimento de insumos técnicos especializados.

\section{Distribuição regional das incubadoras brasileiras}

De 1988 até 2000, houve intenso crescimento do número de incubadoras de base tecnológica no Brasil, que evoluiu de 2 para 76 (ANPROTEC, 2000) ${ }^{5}$. Porém, o processo de crescimento ocorreu de forma concentrada, pois a distribuição regional das incubadoras brasileiras tendeu a refletir a desigual infra-estrutura de ciência e tecnologia do Brasil. A presença de instituições de ensino e pesquisa é fundamental ao surgimento de empresas de base tecnológica porque são fonte de economias externas mediante provisāo de conhecimento, transferência de tecnologia, spin-offs $s^{6}$, assistência técnica e atração de outras empresas (LUGER 8 GOLDSTEIN, 1991).

A Tabela 2 mostra a distribuição percentual por estados e macrorregiões de alguns indicadores de ciência e tecnologia que se relacionam com a capacidade de geração de firmas inovativas. As regiões Sudeste e Sul possuem cerca de $78 \%$ e $83 \%$ do total de instituições de pós-graduação e docentes do país, o que é coerente com a presença de $85 \%$ das incubadoras brasileiras nestas duas macrorregiões.

\footnotetext{
${ }^{5}$ Segundo a ANPROTEC (2000), 56\% do total de 135 incubadoras do país em 2000 podiam ser consideradas de base tecnológica. O restante constitui incubadoras tradicionais e mistas.

${ }^{6}$ Nome dado ao processo de geração de novas empresas a partir de outras já existentes ou de pesquisadores universitários.
} 
Tabela 2: Distribuição percentual de instituiçōes de pós-graduação, docentes permanentes, alunos matriculados, publicações e incubadoras no Brasil - 1999

\begin{tabular}{|c|c|c|c|c|c|c|c|}
\hline \multirow{2}{*}{ Estados } & \multirow{2}{*}{$\begin{array}{l}\text { Instituições de } \\
\text { Pós-Graduaçāo' }\end{array}$} & \multirow{2}{*}{$\begin{array}{c}\text { Docentes } \\
\text { permanentes }\end{array}$} & \multicolumn{2}{|c|}{ Alunos matriculados ${ }^{3}$} & \multirow{2}{*}{$\begin{array}{c}\text { Alunos } \\
\text { matriculados } 4 \\
\text { Graduacão }\end{array}$} & \multirow{2}{*}{ Publicações 5} & \multirow{2}{*}{ Incubadoras 6} \\
\hline & & & Mestrado & Doutorado & & & \\
\hline$A C$ & 0,00 & 0,00 & 0,00 & 0,00 & 0,20 & 0,04 & 0,00 \\
\hline$A M$ & 0,68 & 0,53 & 0,67 & 0,44 & 1,00 & 1,01 & 0,00 \\
\hline $\mathrm{AP}$ & 0,00 & 0,00 & 0,00 & 0,00 & 0,10 & 0,00 & 1,00 \\
\hline PA & 1,44 & 1,02 & 1,41 & 0,52 & 1,80 & 1,17 & 1,00 \\
\hline RO & 0,00 & 0,00 & 0,00 & 0,00 & 0,20 & 0,04 & 0,00 \\
\hline RR & 0,00 & 0,00 & 0,00 & 0,00 & 0,20 & 0,12 & 0,00 \\
\hline TO & 0,00 & 0,00 & 0,00 & 0,00 & 0,30 & 0,04 & 0,00 \\
\hline Norte & 2,12 & 1,55 & 2,09 & 0,95 & 3,90 & 2,40 & 2,00 \\
\hline $\mathrm{AL}$ & 0,48 & 0,38 & 0,23 & 0,03 & 0,70 & 0,35 & 0,00 \\
\hline $\mathrm{BA}$ & 2,40 & 1,57 & 1,41 & 0,61 & 2,80 & 1,58 & 4,00 \\
\hline $\mathrm{CE}$ & 2,46 & 2,03 & 2,05 & 1,06 & 2,20 & 1,72 & 4,00 \\
\hline MA & 0,27 & 0,15 & 0,29 & 0,00 & 1,00 & 0,20 & 0,00 \\
\hline $\mathrm{PB}$ & 2,60 & 2,32 & 2,72 & 0,66 & 1,60 & 1,09 & 2,00 \\
\hline PE & 4,04 & 3,42 & 2,80 & 1,46 & 3,80 & 2,47 & 1,00 \\
\hline $\mathrm{PI}$ & 0,21 & 0,08 & 0,06 & 0,00 & 1,00 & 0,18 & 0,00 \\
\hline RN & 1,78 & 1,39 & 1,46 & 0,39 & 1,20 & 1,09 & 2,00 \\
\hline SE & 0,21 & 0,15 & 0,00 & 0.00 & 0,50 & 0,13 & 0,00 \\
\hline Nordeste & 14,44 & 11,50 & 11,02 & 4,20 & 14,80 & 8,80 & 13,00 \\
\hline$M G$ & 9,31 & 8,71 & 10.68 & 9,71 & 10,60 & 9,90 & 14,00 \\
\hline ES & 0,82 & 0,54 & 1,00 & 0,33 & 1,00 & 0,78 & 1,00 \\
\hline RJ & 15,95 & 16,67 & 15,98 & 18,63 & 9,90 & 17,23 & 8,00 \\
\hline $\mathrm{SP}$ & 34,29 & 39,74 & 35,35 & 49,50 & 32,20 & 41,85 & 32,00 \\
\hline Sudeste & 60,37 & 65,66 & 63,02 & 78,17 & 53,80 & 69,77 & 55,00 \\
\hline $\mathrm{PR}$ & 5,00 & 4,12 & 4,57 & 2,31 & 7,20 & 4,81 & 5,00 \\
\hline RS & 9,51 & 9,18 & 8,98 & 6,79 & 9,30 & 6,64 & 19,00 \\
\hline $\mathrm{SC}$ & 3,22 & 3,45 & 6,62 & 5,77 & 3,80 & 2,85 & 6,00 \\
\hline Sul & 17,73 & 16,75 & 20,17 & 14,87 & 20,30 & 14,30 & 30,00 \\
\hline DF & 3,22 & 2,98 & 2,25 & 1,81 & 1,90 & 2,93 & 1,00 \\
\hline $\mathrm{GO}$ & 1,23 & 0,88 & 0,85 & 0,00 & 2,50 & 0,92 & 0,00 \\
\hline MS & 0,55 & 0,35 & 0,31 & 0,00 & 1,50 & 0,66 & 0,00 \\
\hline MT & 0,34 & 0,33 & 0,29 & 0,00 & 1,40 & 0,22 & 0,00 \\
\hline $\begin{array}{l}\text { Centro- } \\
\text { Oeste }\end{array}$ & 5,34 & 4,54 & 3,71 & 1,81 & 7,20 & 4,73 & 1,00 \\
\hline Brasil & 100 & 100 & 100 & 100 & 100 & 100 & 100 \\
\hline
\end{tabular}

1 Número de instituiçōes que possuem cursos de mestrado e doutorado.

2 Refere-se ao total de docentes permanentes dos cursos de mestrado e doutorado.

3 Alunos Matriculados em Áreas de Formaçāo Tecnológica: Ciências Exatas e da Terra, Computação/Informática, Ciências Biológicas, Engenharias e Ciências Agrárias.

4 Alunos Matriculados em Áreas de Formação Tecnológica, segundo o MEC, em 1998.

5 Refere-se ao número de artigos cientificos publicados.

6 Refere-se ao número total de incubadoras, não havendo desagregaçāo entre as de base tecnológica, tradicionais e mistas.

Fonte: CAPES, MEC, Institute for Scientific Information (ISI) e ANPRO'TEC.

As instituiçōes de pós-graduação e os docentes permanentes dos cursos de mestrado e doutorado podem sustentar o processo de criação de empresas de base tecnológica, uma vez que produzem pesquisas científicas passíveis de serem transformadas em produtos e serviços e emprestam recursos materiais e humanos às empresas recém criadas. Como ressaltam DINIZ \& GONÇALVES (2000), os produtos e processos do novo paradigma tecnológico são intensivos em conhecimento e informação, fazendo com que este tipo de indústria tenda a se localizar 
e consolidar nas regiões de melhor infra-estrutura científica e tecnológica.

A importância da presença de alunos matriculados em cursos de mestrado e doutorado de áreas de formação tecnológica (Ciências Exatas e da Terra, Computação/Informática, Ciências Biológicas, Engenharias e Ciências Agrárias) está relacionada à origem de muitas empresas de base tecnológica, as quais são muitas vezes criadas a partir de dissertações de mestrado ou teses de doutorado. Além dos alunos e de docentes das instituições de pós-graduação, os empreendedores mais comuns são pesquisadores de centros de $\mathrm{P} \mathscr{} \mathrm{D}$ públicos e privados e recém graduados dos cursos mais vinculados às novas tecnologias, como informática e eletrônica.

Cerca de $84 \%$ dos alunos dos cursos de mestrado, 93\% dos alunos de doutorado e $74 \%$ dos alunos de cursos de graduação das áreas de formação tecnológica estão concentrados no Sudeste e Sul do Brasil, dificultando o processo de desconcentração regional de incubadoras e empresas de base tecnológica no país. Do total de alunos dos cursos de doutorado do Brasil, metade está em São Paulo. O número de publicações, que é um indicador da produção de conhecimento científico, revela padrão de concentração semelhante às demais estatísticas, uma vez que apenas o estado de Sāo Paulo responde por $42 \%$ dos artigos publicados.

\section{Desafios e estratégias para o fortalecimento do processo de incuba- ção de empresas}

O processo de criação de empresas inovativas e o sucesso das empresas egressas das incubadoras podem ser dificultados por obstáculos externos e internos ao ambiente de incubação.

Como obstáculos da primeira categoria podem ser citados:

1) conjuntura econômica desfavorável;

2) ausência de políticas governamentais de apoio ao sistema de incubação de empresas;

3) escassez de linhas de financiamento adaptadas às peculiarida. des das empresas de base tecnológica;

4) incerteza técnica do processo de inovação e elevados custos fixos da PED;

5) pouca visibilidade no mercado ou imagem negativa de inexperiência quando a empresa sai da incubadora. 
Como fatores internos às empresas e ao processo de incubação existem:

1) a ineficácia do aprendizado durante o processo de incubação devido ao empreendedor ou à oferta num grau inadequado de algum serviço da incubadora;

2) a avaliação errada do futuro mercado da empresa, desatenção às necessidades de clientes e não antecipação das tendências de mercado para explorar oportunidades tecnológicas;

3) o não envolvimento com pesquisadores externos à empresa que possam colaborar de maneira (in)formal no processo de PED e a ausência de construção de redes de cooperação para inovação, nas quais cada agente tem seu papel definido. As empresas devem ter o objetivo de usufruir do learning by interacting;

4) a desconsideração de outras fontes de informação importantes para inovação, como outras empresas pertencentes à incubadora ou não, concorrentes, clientes e consumidores, empresas de consultoria, fornecedores de bens de capital, universidades e instituições de pesquisa, patentes e licenças, seminários, feiras e publicações.

Embora existam obstáculos que estejam fora do alcance dos gerentes das incubadoras ou dos empresários, como uma conjuntura econômica desfavorável, podem ser traçadas algumas diretrizes para minimizar os efeitos das restrições acima ou mesmo evitá-las.

O Estado pode utilizar seu poder de compra para favorecer as empresas de base tecnológica, reservando parte do orçamento para encomendar produtos com elevado conteúdo tecnológico de empresas nacionais. Legislação específica poderia especificar os percentuais que agências governamentais teriam de reservar para comprar de pequenas empresas e determinar taxas de juros similares às existentes em países desenvolvidos para investimentos em empresas de base tecnológica?

Outra medida seria a criação de incentivos fiscais a pessoas físicas que contribuíssem para formação do "capital semente" de empresas incubadas, além de aumentar o apoio financeiro às incubadoras que selecionam criteriosamente as empresas de base tecnológica, verificando o grau de qualificação da equipe do projeto e/ou a existência de apoio

\footnotetext{
${ }^{7}$ A nova Lei de Inovação prevê a possibilidade de compras da União sem obedecer a lei de licitações. A nova legislaçāo, ao incluir o critério da qualidade tecnológica do produto ou serviço, pode resolver o problema das pequenas empresas que nem sempre conseguem oferecer seus produtos a preços competitivos (LEl..., 2001).
} 
formal e informal de pesquisadores universitários ou de outras instituições de pesquisa com titulação de doutorado. Além disso, o capital de risco deve ser estimulado via incentivos fiscais e legislações que garantam liquidez a este mercado porque é uma forma adequada de financiamento de empreendimentos de base tecnológica.

Outras iniciativas devem ser tomadas pela incubadora e pelos próprios empreendedores. A imagem negativa de amadorismo ou inexperiência, que muitas vezes acompanha as empresas de incubadoras, pode ser solucionada por uma ampliação da visibilidade das empresas, destacando melhor o papel da universidade e da incubadora (LEMOS \& MACULAN, 1998).

A incerteza técnica da inovação pode ser enfrentada por meio do envolvimento, de maneira formal e/ou informal, de pesquisadores com elevada titulação na equipe que realiza o processo de PED. Se isso ocorre, a empresa estará mais próxima do objetivo de monitorar constantemente a evolução dos princípios tecno-científicos. Além da redução dos custos de realização de um processo contínuo de P\&D, maiores serão as chances de resolução de gargalos técnicos nas primeiras fases do projeto e de colocar no mercado um produto/processo com maior conteúdo tecnológico. Se possível, a condução de mais de um projeto de $\mathrm{P} \mathscr{} \mathrm{D}$, que busque inovações incrementais, defensivas ou imitadoras e que tenha interface com o principal produto, ou mesmo a produção de bens com menor conteúdo tecnológico podem diminuir a incerteza técnica e ao mesmo tempo gerar fluxo de caixa que pode ser reempregado no projeto de inovação principal da empresa.

Os gastos em P\&D, apesar de necessários ao processo de capacitação tecnológica da empresa, não podem ser a única fonte de informação para a inovação. A atenção adequada às outras fontes de informação para inovação pode sugerir novos projetos ou mesmo contribuir para o término dos já iniciados, como o uso de instalaçōes e bibliotecas de universidades, o acesso a publicações científicas, a presença em seminários e feiras tecnológicas, as necessidades dos clientes e as parcerias com outras empresas, evitando as fontes que requerem dispêndios adicionais, como a contratação de empresas de consultoria.

Como atuam em nichos de mercado muito especializados, as empresas devem oferecer treinamento ao consumidor do produto com o objetivo de criar demanda para seus produtos, atuando de forma ativa na inovação, no sentido schumpeteriano. Tratamento diferencial aos clientes e atenção às necessidades insatisfeitas constituem fontes de informação importantes para inovações posteriores, tendo em vista o curto 
ciclo de vida dos produtos vinculados às novas tecnologias. Para avaliar a aceitação de seu produto pelo mercado, as empresas podem também reunir potenciais clientes e apresentar o projeto antes de entrarem na incubadora ${ }^{8}$.

\section{Considerações finais}

As incubadoras fornecem assistência às empresas de base tecnológica que reduz parte dos obstáculos e incerteza do processo inovativo. No Brasil, as empresas são criadas por empreendedores com formação em áreas relativas à engenharia e à informática, na maior parte dos casos. Quanto à titulação, é significativa a proporção de mestres e doutores, enquanto outros empresários dispõem de colaboração de pesquisadores externos com título de doutor. Os produtos e serviços desenvolvidos possuem como principal força competitiva o fato de serem diferenciados, sem similar no mercado, ou serem melhor adaptados a aplicações específicas. Isto é coerente com as estratégias de inovação adotadas pelas empresas, que podem variar, em sua maioria, entre ofensivas, defensivas e dependentes.

Há concentração de incubadoras e empresas de base tecnológica nas Regiōes Sudeste e Sul, o que reflete a distribuição desigual de indicadores de ciência e tecnologia como instituição de pós-graduação, docentes, alunos com formação em áreas tecnológicas e publicações.

O fortalecimento do processo de incubação de empresas no Brasil depende da solução de potenciais problemas principalmente relacionados ao financiamento, à incerteza técnica e quanto ao mercado consumidor do produto inovador, à oferta inadequada de serviços pelas incubadoras, à não construção de redes de colaboração com pesquisadores externos e ao não aproveitamento de outras fontes de informação para inovação. O governo pode apoiar mediante linhas de financiamento, incentivos fiscais, legislação específica para empresas de base tecnológica e uso do poder de compra estatal.

Como desdobramentos futuros de pesquisa, pode-se aperfeiçoar o conjunto de informações sobre o tema desse artigo abordando os seguintes pontos: 1) impacto econômico do aumento do número de

\footnotetext{
${ }^{8}$ Isso ocorreu com a KSR, produtora de softwares para engenharia de manufaturas, que reuniu potenciais clientes e conseguiu os seus três primeiros contratos que financiaram o projeto na incubadora (BISCARO, 1999).
} 
incubadoras e empresas de base tecnológica no País; 2) trajetória e estratégias das empresas após a fase de incubação; 3) estrutura organizacional e operacional das incubadoras e seu papel no apoio empresarial; e 4) avaliação das empresas de base tecnológica destacando diferenças macrorregionais do processo de incubação, relativas à formação e à titulação do empreendedor, à colaboração externa de pesquisadores, às estratégias competitivas e de inovação e ao apoio dos governos estaduais e municipais.

\section{Referências bibliográficas}

ANPROTEC. Panorama 2000: as incubadoras de empresas no Brasil. Brasília: ANPROTEC, nov., 2000.

BAÊTA, Adelaide M. C. O desafio da criação: uma análise das incubadoras de empresas de base tecnológica. Petrópolis (RJ): Ed. Vozes, 1999.

BISCARO, F. "Sem sair da incubadora Bússola abre filial em São Paulo". Gazeta Mercantil, Belo Horizonte, 7 jul. 1999. Caderno Por Conta Própria, p. 10.

DINIZ, C. C. E GONÇALVES, E. "Possibilidades e tendências locacionais da indústria do conhecimento no Brasil". In: Encontro Nacional de Economia, 28, Campinas. Anais... Campinas: ANPEC, dez., 2000.

DOSI, G. "The nature of the innovative process". In: DOSI, G. et al. (org.), Technical change and economic theory. London: Pinter Publishers, 1988.

FREEMAN, C 8 SOETE, L. The economics of industrial innovation. Cambridge: The MIT Press, 3d., 1997.

HAGEDOORN, J. "Innovation and entrepreneurship: Schumpeter revisited". Industrial and Corporate Change, v. 5, n. 3, p. 883-896, 1996.

"Lei de inovação mudará relação com setor privado". Gazeta Mercantil, Belo Horizonte, 21, 22 e 23 set. 2001. p. A-6.

LEMOS, Marcelo V. 8 MACULAN, A. "Motivações dos empreendedores para ingresso em incubadoras e principais problemas enfrentados nesses ambientes". In: Seminário nacional de parques tecnológicos e incubadoras de empresas, 8, Belo Horizonte. Anais... Belo Horizonte: ANPROTEC, 1998a. p. 342-350

- "As incubadoras e o apoio às pequenas empresas de base tecnológica para superação de suas dificuldades". In: Seminário nacional de parques tecnológicos e incubadoras de empresas, 8, Belo Horizonte. Anais... Belo Horizonte: ANPROTEC, 1998b, p. 351-362

LUGER, Michael I. \&3 GOLDSTEIN, Harvey. Technology in the garden: research parks and regional economic development. Chapel Hill : University of North Carolina, 1991. 
LUNDVALL, B. "Innovation as an interactive process: from user-producer interaction to the national system of innovation". In: DOSI, G. et al. (org.), Technical change and economic theory. London: Pinter Publishers, 1988.

MACULAN, A. \& CARLEIAL, L. "Aprendizado e interações em pequenas empresas selecionadas: uma abordagem de sistemas nacional e regional de inovações". In: Encontro de economia da região Sul, 2, Curitiba. Anais... Curitiba: ANPEC, 1999.

MALECKI, Edward J. Technology and economic development: the dynamics of local, regional, and national change. New York : Longman, 1991.

MEDEIROS, J. A. \& ATAS, Lucília. "Incubadora de empresas: balanço da experiência brasileira". Revista de Administração. São Paulo, v. 30, n. 1, p. 19-31, jan-mar 1995.

MEDEIROS, José A. "Pólos científicos, tecnológicos e de modernização : uma perspectiva brasileira". Revista Tecbahia. Camaçari, v. 11, n. 1, p. 11-25, jan./ abr., 1996.

MONCK, C. S. P. et al. Science parks and the growth of high technology firms. London: Routledge, 1988.

"Mortalidade menor entre as incubadas". Gazeta Mercantil, Belo Horizonte, 5 jul. 2000. Caderno Por Conta Própria, p. 8.

OECD. Technology incubators: nurturing small firms. Paris: OECD, 1997.

SCHUMPETER. J. A. Teoria do desenvolvimento econômico: uma investigação sobre lucros, capital, crédito, juro e o ciclo econômico. São Paulo: Abril Cultural, $1982[1912]$. 